\title{
Weniger Dissens als man meinen könnte. Ergänzungen zu Elmar Tenorths Metakritik
}

\author{
Nils Berkemeyer
}

Online publiziert: 6. März 2020

(C) Der/die Autor(en) 2020

Heinz Elmar Tenorth hat Recht: „Die Debatte über die Handhabung von Leistung und ihre individuelle Zurechnung in Schulen ist deshalb weder durch Nerowskis Beitrag noch Berkemeyers Art von Kritik beendet, sondern bestenfalls eröffnet“" (Tenorth, letzter Absatz vor Literatur). Ebendies in Bezug auf den Beitrag von Nerowski deutlich zu machen, war genau das Anliegen meiner Kritik, die in erster Linie zeigen sollte und gezeigt hat, das Fazit von Tenorth bestätigt dies, dass Nerowskis Versuch der Legitimation des Leistungsprinzips im Schulsystem nicht trägt. Anders als Tenorth behauptet, wollte ich das Leistungsthema damit aber nicht ad acta legen, sondern vielmehr verdeutlichen, dass die Legitimation in Bezug auf das Prinzip und die Verfahren nach wie vor aussteht.

Was in meinem Beitrag zu kurz kommt, ist die Darstellung einer eigenen Position, hier hat Tenorth ebenfallsRecht, wenn er darauf hinweist, dass wesentliche Bezüge an der einen oder anderen Stelle fehlen. Allerdings ist der Fokus meines Beitrags die Kritik am Begründungsversuch des Leistungsprinzips, nicht die Entwicklung einer eignen Position. Dies mag man bedauern, aber es ist etwas irreführend, dauernd auf das Fehlen von Inhalten hinzuweisen, deren Darstellung gar nicht dem Anliegen eines Autors entsprechen. Dies scheint mir das erste Missverständnis der Metakritik zu sein.

Ein zweites Missverständnis liegt in Tenorths Auffassung meiner Behauptung, Nerowski versuche etwas zu legitimieren, was bereits legitimiert ist und dies mit einer Idee, die Walzer bereits 1983 sehr ähnlich formuliert hat. Die von Tenorth angeführten kritischen Bemerkungen zeigen letztlich nur, dass es häufig um die richtigen Verfahren und Begründungen Leistung zu legitimieren gerungen wird, das

Prof. Dr. N. Berkemeyer $(\bowtie)$

Lehrstuhl für Schulpädagogik und Schulentwicklung, Friedrich-Schiller Universität Jena,

Fürstengraben 11, 07743 Jena, Deutschland

E-Mail: nils.berkemeyer@uni-jena.de 
Leistungsprinzip selbst aber nicht in Zweifel gezogen wird. Darum muss es auch nicht neu legitimiert werden. Zudem sieht wohl auch Tenorth im Beitrag Nerowskis keine Weiterentwicklung der Position von Walzer, teilt aber meine Einschätzung, dass sich die Ansätze sehr ähneln. Es mag also aus dramaturgischen Gründen verständlich sein, sich für die Rettung des Leistungsprinzips vor den unverbesserlichen Träumern der pädagogischen Provinz zu gerieren (so die Anspielungen bei Tenorth), allerdings ändert diese Form der Rhetorik nichts daran, dass das Leistungsprinzip sich breiter Anerkennung erfreut, institutionell im Schulsystem verankert ist und von zahlreichen Erziehungswissenschaftlern und -wissenschaftlerinnen vertreten wird; auch von so prominenten wie Tenorth, wie man am Ende des Beitrags erfahren kann. Die Debatte sollte sich darum weniger auf bildungspolitische Positionierungen kaprizieren, sondern auf eine einfache akademische Frage konzentrieren: Ist es, und wenn ja, wie ist es möglich, Leistung im Schulsystem gerechtigkeitstheoretisch zu legitimieren.

Tenorths Metakritik verweist nun auf drei zentrale Probleme meiner Kritik: „Zuerst den Vorwurf, ein Problem zu bearbeiten, das längst gelöst ist, [...] [m]eine Behauptung, Nerowski habe die Diskussionslage falsch, verkürzt und nicht problemangemessen rezipiert, und, [...] drittens, für [m]eine Kritik an den schultheoretischen Annahmen in Nerowskis Analyse“ (Tenorth, erste Manuskriptseite).

Der erste Punkt der Metakritik erklärt sich aus dem Missverständnis wie ich es oben beschrieben habe. Es ist gut, dass Walzer ausführlicher zur Darstellung gelangt und so deutlich wird, dass Nerowskis Ansatz sich von Walzer kaum unterscheidet, man könnte ihn auch als Dublette bezeichnen. Es stimmt zudem, dass ein Beitrag zu Walzer dem von mir mitherausgegeben Band über Gerechtigkeit im Schulsystem fehlt (Manitius et al. 2015). Nur diese Nachlässigkeit kann wohl kein Argument dafür sein, so rhetorisch klug dieser Hinweis platziert ist, dass Nerowski einen weltweit bedeutsamen gerechtigkeitstheoretischen Ansatz unterschlägt, der mit seinem nahezu identisch ist. Egal wie sehr Tenorth auf meine Rezeptionslücken hinweist, im Sinne der Aufarbeitung des Forschungsstandes wäre Walzer ein zwingender Ausgangspunkt für Nerowskis Argumentation gewesen. Denn nur so kann man sehen, dass erstens das gerechtigkeitstheoretische Problem der Legitimation des Leistungsprinzips in Schule nicht gelöst wird und Leistung auch nicht als Legitimationsprinzip gerettet werden muss. Genau in diesem Sinne hat Nerowski Probleme konstruiert, die in dieser Form gar nicht existieren.

Der zweite Punkt ist interessant, denn Tenorth will ihn ,nicht weiter im Detail diskutieren“ (Tenorth, S. 4 Manuskript). Es geht um meine kritischen Einwände in Bezug auf spezifische Lesarten. Es geht mir aber nicht um Lesarten, sondern um die Konstruktion von Begründungen. Wer Autoren ins Feld führt, die nachweislich (und eben nicht je nach Lesart) nicht für die gewählte Argumentation taugen, dies gilt eindeutig für Wigger, Taylor und Miller, der muss mit Kritik und Widerspruch, nicht nur mit einer ergänzenden Deutung, rechnen.

Der dritte Punkt bezieht sich auf die schultheoretischen Deutungen Nerowskis im Anschluss an Miller sowie die Kritik am Anerkennungsdiskurs. Zunächst ist Tenorth zuzustimmen, dass Kriterien der Gerechtigkeit allein für den Diskurs über schulsystemische Gerechtigkeit nicht genügen, sondern auch die Praktiken, oder, wie ich formuliert habe, Verfahren, in Rechnung gestellt werden müssen. Auch hier 
liegt also eine Gemeinsamkeit in der Kritik. Richtig ist auch, dass meine Kritik den Anerkennungsdiskurs in seiner Breite nicht umfasst. Ich habe mich zunächst darauf beschränkt, zu zeigen, dass Nerowskis Darstellung von Honneth unzureichend ist. Dies gilt nach wie vor, weil auch Tenorth nicht entkräften kann, dass es deutliche Ähnlichkeiten zwischen Honneth und Miller gibt (die bei Nerowski verdeckt bleiben und so der Eindruck zweier geradezu entgegengesetzter Positionen entsteht) und weil auch Tenorth auf die Sozialtheorie Honneths nicht eingeht, sondern sie lediglich mit Blick auf ihre Kritiker und Kritikerinnen zu diskreditieren versucht. Dies ist handelsüblich, erklärt aber nicht, warum selbst Honneth vermutlich mit dem Leistungsprinzip in Schule leben könnte, Nerowski und Tenorth aber schlicht seinen Ansatz ablehnen oder diesen wichtigen Punkt übersehen. Ich habe diesbezüglich argumentiert, dass Honneth zu stark interaktionistisch rezipiert wird und sein sozialtheoretischer Ansatz in der Rezeption weitgehend ungesehen bleibt. Darum könnte man vermutlich eher mit Honneth als mit Nerowski, Tenorth oder Miller das Leistungsprinzip im Schulkontext zu rechtfertigen versuchen. In Bezug auf Miller bleibt meine Kritik von Tenorth im Grunde unberührt. Tenorth sieht wohl auch, dass das Äquivalenzprinzip, welches für Miller konstitutiv für das Leistungsprinzip ist, im Schulsystem nicht erfüllt ist und auch er - wie ich - kann sich wohl mit der Vorstellung, Schule ab Klasse 9 als Zweckverband zu konzipieren, nicht anfreunden. Tenorth möchte zwar wie Nerowski das Leistungsprinzip stark machen, aber Nerowskis Argumente - und hier liegt die entscheidende Gemeinsamkeit - können Tenorth letztlich nicht überzeugen (wenn womöglich auch aus anderen Gründen), weshalb die Debatte selbstredend nicht ,beendet, sondern bestenfalls eröffnet [ist]“ (Tenorth, letzter Satz).

Was am Ende der Diskussion aus meiner Sicht bleibt, ist, dass folgende Argumente meiner Kritik nicht widerlegt sind und somit (vorerst) weiterhin Gültigkeit beanspruchen können:

- Die Position von Walzer wird nicht aufgegriffen und weiterentwickelt, dies sollte künftig Berücksichtigung finden (so auch Tenorth)

- Die Kritik in Bezug auf die Verwendung von Autoren wie Wigger, Taylor und Miller für die Zwecke der Argumentation von Nerowski bleibt bestehen. Nicht nur stützen sie Nerowskis Position nicht, sie lassen sich eher für Gegenargumente verwenden, wie ich dies unter Verwendung von Textbelegen gezeigt habe.

- Da Nerowski das Äquivalenzprinzip, konstitutives Merkmal für das Leistungsprinzip bei Miller, nicht berücksichtigt, kann seine schultheoretische Deutung Millers in dieser Form keine Gültigkeit beanspruchen (Tenorth widerspricht hier nicht).

- Das Potential von Honneths Sozialtheorie für die Legitimation des Leistungsprinzips in Schule wird weder von Nerowski noch von Tenorth gesehen. Honneth ist nicht zwingend der Gewährsmann für die Abschaffung des Leistungsprinzips in der Schule.

- Insgesamt kann Nerowskis Ansatz nicht als geeignete Begründung für das Leistungsprinzip im Schulsystem betrachtet werden (so auch Tenorth).

- Eine gerechtigkeitstheoretische Ergänzung des schultheoretischen Diskurses ist nötig und weiterführend (so auch Tenorth). 
Es wäre wünschenswert, wenn diese Debatte dazu beitragen könnte, gerechtigkeitstheoretische Positionen systematisch in den Diskurs einzubeziehen und mit pädagogischen Positionen und schultheoretischen Überlegungen $\mathrm{zu}$ verbinden. Einen Versuch hierzu habe ich andernorts unternommen (Berkemeyer 2019).

Funding Open Access funding provided by Projekt DEAL.

Open Access Dieser Artikel wird unter der Creative Commons Namensnennung 4.0 International Lizenz veröffentlicht, welche die Nutzung, Vervielfältigung, Bearbeitung, Verbreitung und Wiedergabe in jeglichem Medium und Format erlaubt, sofern Sie den/die ursprünglichen Autor(en) und die Quelle ordnungsgemäß nennen, einen Link zur Creative Commons Lizenz beifügen und angeben, ob Änderungen vorgenommen wurden.

Die in diesem Artikel enthaltenen Bilder und sonstiges Drittmaterial unterliegen ebenfalls der genannten Creative Commons Lizenz, sofern sich aus der Abbildungslegende nichts anderes ergibt. Sofern das betreffende Material nicht unter der genannten Creative Commons Lizenz steht und die betreffende Handlung nicht nach gesetzlichen Vorschriften erlaubt ist, ist für die oben aufgeführten Weiterverwendungen des Materials die Einwilligung des jeweiligen Rechteinhabers einzuholen.

Weitere Details zur Lizenz entnehmen Sie bitte der Lizenzinformation auf http://creativecommons.org/ licenses/by/4.0/deed.de.

\section{Literatur}

Berkemeyer, N. (2019). Gerechtigkeit als Maßstab für das Schulsystem? In R. Koerrenz \& N. Berkemeyer (Hrsg.), Das Schulsystem auf dem Prüfstand. Weinheim: Beltz Juventa.

Manitius, V., Hermstein, B., Berkemeyer, N. \& Bos, W. (2015). Zur Gerechtigkeit von Schule. Theorien, Konzepte, Analysen. Münster: Waxmann. 